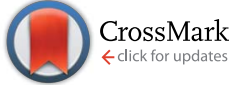

Cite this: RSC Adv., 2017, 7, 11672

\title{
Selection, identification and application of DNA aptamers for the detection of Bifidobacterium breve $\uparrow$
}

\author{
Lujun Hu, ${ }^{a}$ Linlin Wang, ${ }^{a}$ Wenwei Lu, ${ }^{\text {ab }}$ Qixiao Zhai, ${ }^{\text {ab }}$ Daming Fan, ${ }^{\text {ab }}$ Xiaoming Liu, ${ }^{\text {ab }}$ \\ Jianxin Zhao, ${ }^{\text {ab }}$ Hao Zhang ${ }^{\text {ab }}$ and Wei Chen ${ }^{\star a b c}$
}

In the present study, a single-stranded DNA (ssDNA) aptamer binding to Bifidobacterium breve with high avidity and selectivity was selected through a whole-bacterium-based systemic evolution of ligands using an exponential enrichment (SELEX) process. Following 12 rounds of selection specific for B. breve, three FAM-labeled aptamers were chosen for flow cytometry analysis and the results revealed that all three aptamers possessed a high binding affinity for $B$. breve. To obtain the optimal sequence, sequence truncation experiments of these three aptamers were conducted. An aptamer variant BB16-11f with high affinity and selectivity was acquired. In addition, the dissociation constant was significantly reduced to $18.66 \pm 1.41 \mathrm{nM}$. Furthermore, an enzyme linked aptamer assay was developed to prove the potential application of the aptamer BB16-11f in the detection of B. breve. The results showed that the colorimetric assay had a linear relationship between the absorbance at $450 \mathrm{~nm}$ and the concentrations

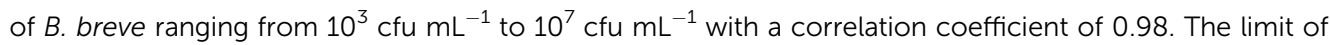

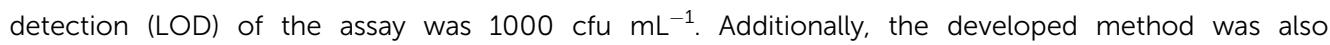
successfully used to detect $B$. breve in a milk environment. Taken together, we hold that the developed colorimetric bioassay based on the aptamer BB16-11f is a promising method for the detection of $B$. breve.

Received 2nd December 2016 Accepted 9th February 2017

DOI: 10.1039/c6ra27672e

rsc.li/rsc-advances the product labels. ${ }^{13-16}$ Thus, it is vital to discriminate the different probiotic strains at the species level in food products available on the market.

Identification of $B$. breve is significant for its industrial use, but the conventional methods of detection do not always obtain satisfying results. ${ }^{17,18}$ The classical culture methods, including isolation and identification of the species, are labor intensive and time consuming. Furthermore, phenotypic characterization is not enough to distinguish $B$. breve from other strains at the species level in many cases. ${ }^{19}$ Thus, there is an important need to develop an alternative approach to the identification of B. breve. Fortunately, various molecular methods have been developed to detect $B$. breve. Molecular techniques such as fluorescence in situ hybridization ${ }^{20}$ and $\mathrm{PCR}^{21}$ can shorten the detection time, but they increase the analysis cost in that they need sophisticated equipment and well-trained professional. ${ }^{21-23}$ What is more, these methods are not practical for rapid detection.

Rapid identification methods should supply dependable, low-cost, real time, on-field and amicable detection with same or better sensitivity, selectivity and consistency of the culturebased assays. ${ }^{24}$ Aptamers, which are screened via an in vitro process, are single-stranded oligonucleotide ligands obtained by SELEX method based on their high binding affinity and selectivity for target biomolecules. ${ }^{25,26}$ Aptamers have many unique features that make them a better choice than 
antibodies, including inexpensive, ease of synthesis and modification and comparable stability. ${ }^{27,28}$ Live bacteria and bacterial surface molecules can be employed as targets during SELEX. ${ }^{29,30}$ Whole bacteria as target has been implemented to create ssDNA aptamers against Mycobacterium tuberculosis, Lactobacillus acidophilus, Staphylococcus aureus, Campylobacter jejuni, Streptococcus pyogenes and Salmonella typhimurium without knowing a specific target molecule beforehand. ${ }^{31-36}$ Thus, aptamers may offer an alternative method for the rapid detection of B. breve.

Enzyme linked aptamer assay (ELAA), is a variant of conventional ELISA (enzyme linked immunosorbent assay) using aptamers replacing antibodies. ELAA can realize colorimetric assay based on the catalytic reaction of horseradish peroxidase (HRP) and TMB- $\mathrm{H}_{2} \mathrm{O}_{2}$. And colorimetric assays have many advantages such as high-throughput with the help of 96well microplate and convenience in that the signal readout requires only simple instruments or even no instrumentation when read with the naked eyes. Thus, ELAA has been used in many bioanalytical applications for the target-specific detection of some substances such as ochratoxin A, thrombin and $M$. tuberculosis. ${ }^{37-39}$ However, ELAA has not been reported in the detection of $B$. breve.

In the study, we used an improved whole-bacterium SELEX strategy to screen ssDNA aptamers with high avidity and selectivity for $B$. breve. Additionally, the truncation experiments were conducted to obtain the optimal aptamer. Furthermore, to confirm the potential application of the candidate aptamer, a colorimetric bioassay was developed for the detection of $B$. breve, and the developed method was also used to detect $B$. breve in milk samples.

\section{Materials and methods}

\section{Bacterial strains and culture media}

$B$. breve ATCC 15700 was employed as the target during wholebacterium SELEX. Other bacterial strains adopted in the study (for counter-SELEX and specificity studies) were as follows: $B$. longum ATCC 15697, B. bifidum ATCC 29521, B. animalis JCM 11658, B. adolescentis ATCC 15705 and L. plantarum ST-III (CGMCC no. 0847). All of the bifidobacterial strains used in the study were supplied by the American Type Culture Collection (ATCC) or the Japan Collection of Microorganisms (JCM). All bifidobacterial strains were grown in MRS broth with addition of $0.05 \%$ of L-cysteine hydrochloride monohydrate at $37{ }^{\circ} \mathrm{C}$. The $L$. plantarum used in this study was cultured in MRS broth at $37{ }^{\circ} \mathrm{C}$ (Merck KGaA, Darmstadt, Germany). All bacterial strains were cultured to the logarithmic phase under anaerobic conditions.

\section{DNA library and PCR amplification}

An 80 nt oligonucleotide ssDNA library was synthesized with the following sequence:

5'-AGCAGCACAGAGGTCAGATG-N40-CCTATGCGTGC-

TACCGTGAA-3', where the middle N40 represents random nucleic acids with equimolar amounts of $\mathrm{A}, \mathrm{G}, \mathrm{C}$ and $\mathrm{T}$ at each position and the fixed primer sequence is at both ends. The initiating ssDNA library and the primers employed to amplify it were supplied by Integrated DNA Technologies (IDT; Coralville, IA).

The PCR amplification methods during SELEX were as follows: $1 \times$ PCR amplification buffer, $10 \mu \mathrm{M}$ forward and

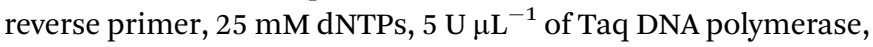
$2 \mu \mathrm{L}$ of the template (all reagents were from Invitrogen) and 2.5 $\mu \mathrm{L}$ of DMSO (Sigma) in a total volume of $50 \mu \mathrm{L}$. Thermocycling parameters were as follows: pre-denaturation for $6 \mathrm{~min}$ at $95^{\circ} \mathrm{C}$, 25 cycles of denaturation at $95{ }^{\circ} \mathrm{C}$ for $30 \mathrm{~s}$, annealing for $30 \mathrm{~s}$ at $69{ }^{\circ} \mathrm{C}$, elongation for $20 \mathrm{~s}$ at $72{ }^{\circ} \mathrm{C}$ and final elongation for $6 \mathrm{~min}$ at $72{ }^{\circ} \mathrm{C}$ (T100 Thermo cycler; Bio-Rad Laboratories, Hercules, CA). PCR amplification products were analyzed by $8 \%$ nondenaturing polyacrylamide gel electrophoresis (PAGE) in $1 \times$ TBE buffer (Bio-Rad Protean III) at $200 \mathrm{~V}$ for $25 \mathrm{~min}$. Qiagen MinElute PCR Purification Kit was used for purifying all PCR amplification products (Qiagen Inc., Valencia, CA).

\section{Aptamer selection}

The SELEX procedure for aptamer selection was based on Hamula and colleagues ${ }^{32}$ with some modifications as follows: $B$. breve was harvested when it reached logarithmic phase. $B$. breve cells were centrifuged at $4000 \times g$ and $4{ }^{\circ} \mathrm{C}$ for $5 \mathrm{~min}$ and then washed twice in binding buffer (50 mM Tris- $\mathrm{HCl}$ (pH 7.4), $100 \mathrm{mM} \mathrm{NaCl}, 5 \mathrm{mM} \mathrm{KCl}$ and $1 \mathrm{mM} \mathrm{MgCl}_{2}$ ). The ssDNA library/ pool was heated at $95{ }^{\circ} \mathrm{C}$ for $10 \mathrm{~min}$ and rapidly chilled for $10 \mathrm{~min}$ in an ice bath before incubation. B. breve cells totaling $10^{8} \mathrm{cfu} \mathrm{\textrm {mL } ^ { - 1 }}$ were incubated with the $10 \mu \mathrm{L}$ ssDNA library (200 $\mu \mathrm{M})$ for initial round or $10 \mu \mathrm{L}$ aptamer pool $(10 \mu \mathrm{M})$ for subsequent rounds in binding buffer $(600 \mu \mathrm{L}$ for the initial round, $350 \mu \mathrm{L}$ for subsequent rounds) for $45 \mathrm{~min}$ at room temperature with slight agitation. An excess of tRNA (Sigma) and bovine serum albumin (BSA; Invitrogen) were put into the incubation buffer. Following incubation, B. breve cells were washed three times in binding buffer (from 0.25 to $0.75 \mathrm{~mL}$ ) with $0.05 \%$ BSA. And then, the cells were resuspended in $80 \mu \mathrm{L}$ of $1 \times$ PCR reaction buffer before next heating at $95{ }^{\circ} \mathrm{C}$ for $10 \mathrm{~min}$ and quickly cooled for $10 \mathrm{~min}$ in an ice bath. After centrifugation as described above, the supernatant was separated and designated as the cell-bound aptamer fraction, which was used as template for PCR amplification and the PCR amplification products were adopted in the following round of selection.

To exclude aptamers binding to the tube, the resuspended cell solution from each incubation and elution was transferred to a fresh tube. To improve the specificity of aptamer candidates, two rounds (the 9th and 11th rounds) of counter-SELEX were implemented. In brief, the selected aptamer pools were incubated with the pooled counter-SELEX bacteria (B. longum, $B$. bifidum, B. animalis and B. adolescentis) in binding buffer for $45 \mathrm{~min}$ at room temperature with gentle rotation. The aptamerbound cells were centrifuged and the supernatant were collected for the next round of selection.

Following the 12th round of selection, aptamer pools were cloned (TOPO TA Cloning kit, Invitrogen, Carlsbad, CA) and the 
plasmid DNA was purified (QIAEX II Gel Extraction Kit; Qiagen, Mississauga, ON, Canada) prior to sequencing. RNA structure 3.0 was adopted for predicting the secondary structure of each sequence, with input conditions of $1 \mathrm{mM} \mathrm{MgCl}$ and the room temperature. The most likely sequence was selected according to the lowest predicted free energy $\left(\Delta G, \mathrm{kcal} \mathrm{mol}^{-1}\right)$.

\section{Binding assays}

A FACSCalibur flow cytometer with PowerMacG4 workstation and CellQuest Pro software (BD Biosciences, USA) was used to analyze the binding affinity of the individual sequences to different bacterial cells (B. breve, B. longum, B. bifidum, B. animalis, $B$. adolescentis and L. plantarum) in separate experiments. The individual sequences with the fluorescent label ( $5^{\prime}$-FAM) attached were obtained from IDT. The binding analysis were performed by incubating $10^{8}$ B. breve cells with $100 \mathrm{nM}$ of FAMlabeled aptamer for $45 \mathrm{~min}$ at room temperature with gentle rotation to facilitate aptamer binding following heat denaturation. Cells were then washed twice before resuspension in binding buffer for prompt flow cytometric assays. Forward scatter, side scatter and fluorescence intensity were measured, and the gated fluorescence intensity above the background (cells with no aptamers) was quantified. BD CellQuest Pro software was used for analyzing the data from the FACSCalibur and creating the histogram overlays. Binding saturation curves were created to estimate binding dissociation constants $\left(K_{\mathrm{d}}\right)$ by altering the aptamer concentration $(0-100 \mathrm{nM})$ with a constant number of $B$. breve cells $\left(10^{8}\right.$ cells). ${ }^{32,40}$ GraphPad Prism 5.0 software was employed to fit a nonlinear regression curve from which $K_{\mathrm{d}}$ values were determined.

\section{Aptamer truncation}

To determine the minimal sequence necessary for high-affinity binding between the aptamers and $B$. breve, truncation experiments were conducted. ${ }^{41}$ Firstly, the specific primer binding sites at both ends of the aptamers were removed. Then, DNA aptamer variants with high binding ability to $B$. breve were further truncated from the $5^{\prime}$ or $3^{\prime}$ end respectively. In the experiment, the truncated aptamer variants were FAM-labeled at the $5^{\prime}$-end and tested for their binding affinity with $B$. breve using flow cytometric assays as described above. And the specificity and binding dissociation constants $K_{\mathrm{d}}$ values of the optimized aptamer variants were also determined according to the method described above.

\section{Procedure of colorimetric bioassay based on the selected aptamer}

A colorimetric sandwich-type assay for detection $B$. breve was developed according to ELAA. First, avidin was dissolved in $\mathrm{NaHCO}_{3}$ buffer (pH 9.6) and $100 \mu \mathrm{L}$ diluted avidin $\left(10 \mathrm{mg} \mathrm{mL}^{-1}\right)$ was added into each well of the 96-well plates for incubating overnight at $4{ }^{\circ} \mathrm{C}$. The wells were washed three times with washing buffer $\left(0.01 \mathrm{~mol} \mathrm{~L}^{-1} \mathrm{PBS}\right.$ with $0.05 \%$ Tween-20) prior to use. Then, the avidin-coated microplate wells were blocked with the blocking buffer ( $0.01 \mathrm{~mol} \mathrm{~L}^{-1}$ PBS with $\left.3 \% \mathrm{BSA}\right)$ to prevent the appearance of nonspecific adsorption.
Subsequently, $10 \mu \mathrm{L}$ biotinylated candidate aptamer was added to the wells of the microplates and incubated $30 \mathrm{~min}$ at $37{ }^{\circ} \mathrm{C}$ after the plates were washed three times with the washing buffer. A series of different concentrations of $B$. breve cells were then added into each well for incubating at $37^{\circ} \mathrm{C}$ for $45 \mathrm{~min}$ and the biotinylated aptamer and streptavidin-HRP were mixed at $37{ }^{\circ} \mathrm{C}$ for $30 \mathrm{~min}$ at the same time. After the microplates were washed three times with the washing buffer, $10 \mu \mathrm{L}$ samples of the above biotinylated aptamer and streptavidin-HRP complexes were added to the wells of the microplates for reacting for $30 \mathrm{~min}$ at $37{ }^{\circ} \mathrm{C}$. Then $200 \mu \mathrm{L}$ TMB- $\mathrm{H}_{2} \mathrm{O}_{2}$ working solutions were added into each well for reacting without direct light exposure after the final wash step. After $15 \mathrm{~min}$, the mixture was terminated with $50 \mu \mathrm{L} 2 \mathrm{M} \mathrm{H}_{2} \mathrm{SO}_{4}$, and the OD value at $450 \mathrm{~nm}$ was measured with the microplate reader.

\section{Colorimetric detection of $B$. breve in the milk}

Raw milk was ordered from local supermarkets and subjected to centrifugation for $30 \mathrm{~min}$ at $14000 \mathrm{rpm}$ so that protein and fat were removed. Then, the supernatant was filtered with a 0.22 $\mu \mathrm{M}$ filtration membrane. Finally, different amounts of $B$. breve were added into the treated milk samples to make the spiked samples for colorimetric detection as described above. The plate counting method was also used for detecting $B$. breve as a control test in the study.

\section{Results and discussion}

\section{Aptamer selection}

To screen aptamers that potentially identify $B$. breve, a wholebacterium SELEX including 10 rounds of selection against $B$. breve and two rounds of counter selection against an incorporative mixture of other bacteria was performed. The wholebacterium SELEX approach has two advantages for the rapid identification of $B$. breve. First, it is unnecessary to be aware of the target beforehand and the target is in its native conformation, which inherently increases the possibility of intended application. ${ }^{42}$ Second, the whole-bacterium SELEX approach is also convenient for both separation based on centrifugation and analysis of binding affinity via flow cytometry. ${ }^{31,43}$

The aptamer pools from the 12th round of SELEX were cloned and sequenced, obtaining 33 sequences totally (data shown in Table S1 in the ESI†). These sequences were then divided into six families on the basis of the homology of the DNA sequence and the similarity of the secondary structure. Analysis of all sequences revealed that many sequences contain high GC content, which indicates the secondary structure formation. Sequences were selected for further characterization according to their predicted secondary structure and free energy of formation.

\section{Determination of affinity and specificity}

The FAM-labeled aptamers were incubated with $B$. breve and were then tested via flow cytometry. The results showed that the values of gated fluorescence intensity above the background of the three aptamers BB2p, BB10p and BB16p were approximately 
$74 \%, 68 \%$ and $77 \%$ respectively, which were from the aptamer pool of the 12th round of SELEX. These three aptamer sequences are shown in Table 1. Aptamer BB16p showed the greatest binding affinity with $B$. breve. In addition, Fig. 1 predicted the secondary structures of the aptamers, and the conditions input were $1 \mathrm{mM} \mathrm{MgCl}_{2}$ and the room temperature.

Fluorescently labeled aptamers BB2p, BB10p and BB16p were analyzed against different species of bacteria, including
B. longum, B. bifidum, B. animalis, B. adolescentis and L. plantarum. These three aptamers all showed higher binding affinity for $B$. breve over the other species of bacteria. Although the percent gated fluorescence intensity above the background was $11.2 \%$ for BB10p when incubated with B. bifidum (Fig. 2A and B), the aptamers BB2p and BB16p showed lower binding to $B$. bifidum with the values of gated fluorescence intensity above the background $8.9 \%$ and $7.0 \%$ respectively

Table 1 Tested full-length aptamer sequences $(80 \mathrm{nt})^{a}$

\begin{tabular}{ll}
\hline Name & Sequence $\left(5^{\prime} \rightarrow 3^{\prime}\right)$ \\
\hline BB2p & $\underline{\text { AGCAGCACAGAGGTCAGATG CCGGGCAGCGGTCAATGCCGCACCTTCCATATGATCGGGG } \underline{\text { CCTATGCGTGCTACCGTGAA }}}$ \\
BB10p & AGCAGCACAGAGGTCAGATG GGCCCCCTGCCTGCCAAAAAGGTGTTGCCAGGTTGGCGGC CCTATGCGTGCTACCGTGAA
\end{tabular}

BB16p

AGCAGCACAGAGGTCAGATG CTCCCAGGCCGTTGGGGCGTTGCCTGCGTGCACCCGGGGC CCTATGCGTGCTACCGTGAA

${ }^{a}$ The underlined sequences are the primer binding sites.

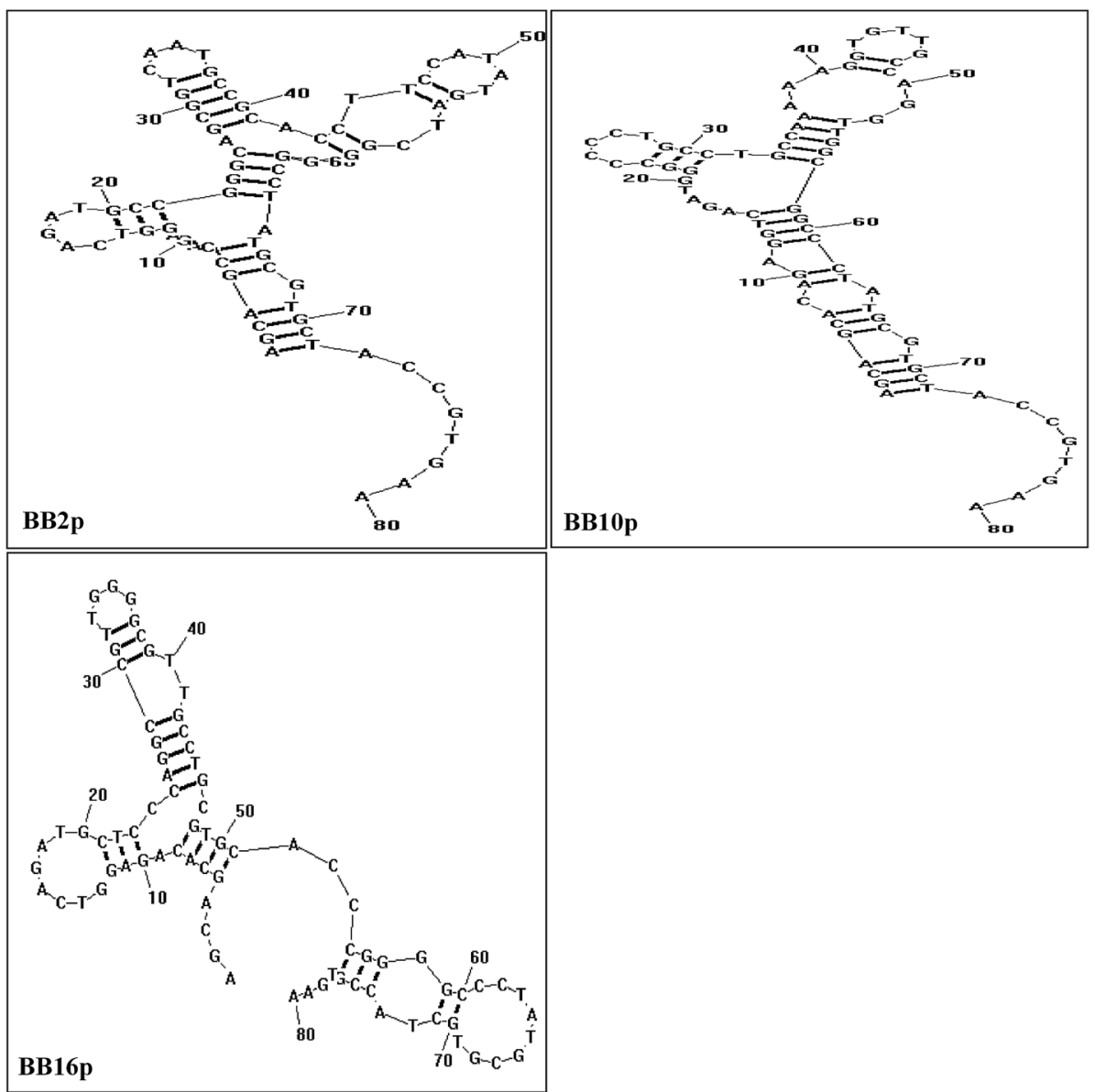

Fig. 1 Predicted secondary structures of aptamer sequences that have high binding avidity for $B$. breve in SELEX. The conditions used were $1 \mathrm{mM}$ $\mathrm{MgCl}_{2}$ and the room temperature. 

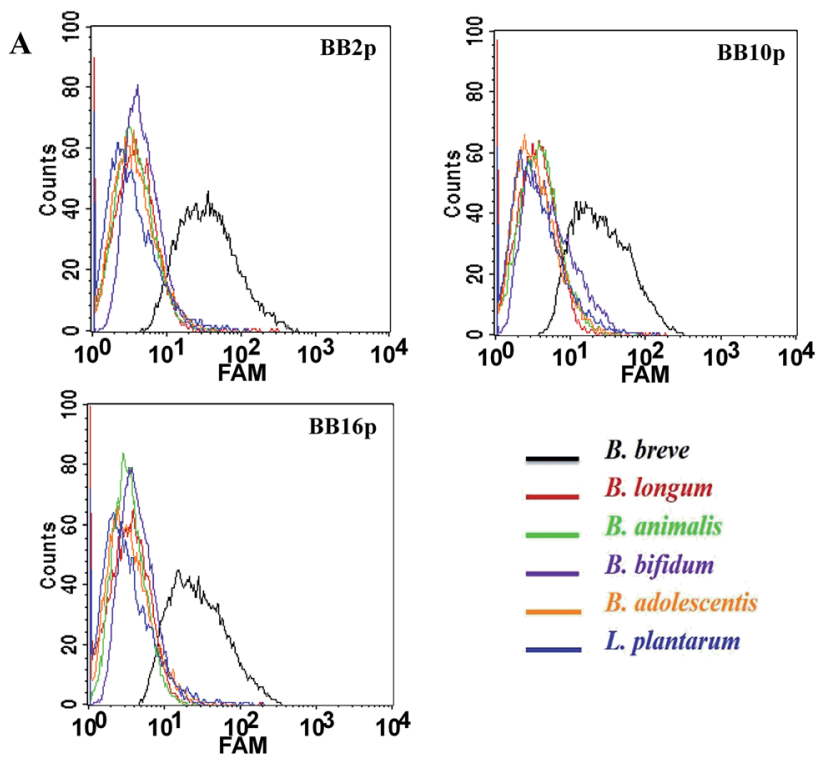

B

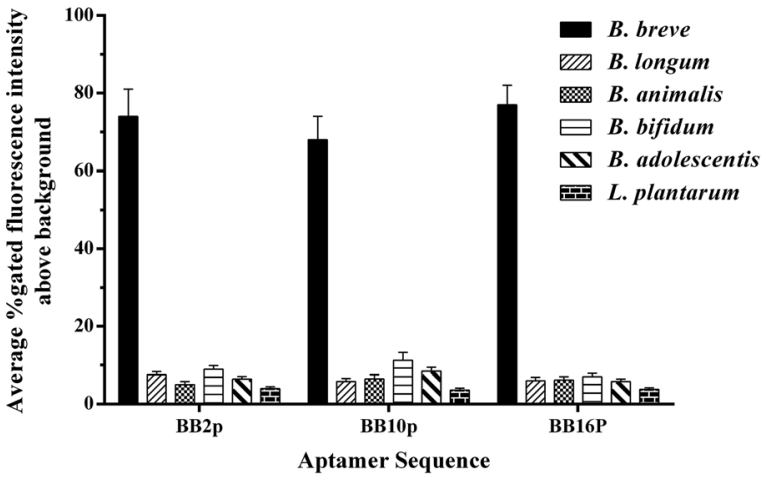

Fig. 2 Binding specificity of individual aptamers for $B$. breve. The fluorescently labeled individual aptamers were incubated with different species of bacteria as described in text. (A) Flow cytometric assays of individual aptamers binding ability to different species of bacteria. Different species of bacteria is shown with differently colored curves. (B) Histogram of the percent gated fluorescence intensity above the background for individual aptamers.

(Fig. 2A and B). Thus, it can be inferred that the aptamers $\mathrm{BB} 2 \mathrm{p}$ and $\mathrm{BB} 16 \mathrm{p}$ are more specific for $B$. breve than the aptamer BB10p.

\section{Aptamer optimization}

Full-length aptamers, being with fixed primer sequences at each terminus for PCR amplification, were selected through the SELEX process. In general, not all the nucleic acids are necessary for the binding affinity between the aptamers and the targets. ${ }^{44}$ And longer sequences bring about lower yield and higher cost in synthesis. Moreover, the unnecessary nucleic acids could form intricate secondary structures that destabilize the binding affinity between the aptamers and the targets. ${ }^{41}$ Thus, in practical usage, it is necessary to obtain the minimal sequence of aptamers which showed the same or higher affinity to the target compared to the full-length aptamer.

To obtain the minimal sequence of aptamers, the aptamers BB2p, BB10p and BB16p were truncated to narrow down the sequence region responsible for target binding. All the truncated aptamer variants were shown in Table 2. When the primers of the aptamers were removed, the aptamer variants BB10 and BB16 demonstrated higher binding affinity than the original full-length aptamers respectively, while aptamer variant BB2 revealed lower binding affinity compared to the aptamer BB2p (Fig. 3). Therefore, the results proved that the primer binding sites of BB2p were very important for its binding affinity for the target, while the primer sequences of BB10p and BB16p were unnecessary for their binding abilities to B. breve.

The aptamer variants BB10 and BB16 were further stepwise truncated 6-15 nt from $3^{\prime}$-end or $5^{\prime}$-end respectively. In other words, when the nucleotides were removed from $3^{\prime}$-end of the aptamer variants, $5^{\prime}$-end remained intact bringing about the further aptamer variants BB10-6f, BB10-10f, BB10-14f, BB16-11f, BB16-13f and BB16-15f, and vice versa, when the sequences of $3^{\prime}-$ end were removed resulting in the further aptamer variants BB10-2r, BB10-9r, BB10-13r and BB16-8r. In these ten further truncated variants, one or more G-clusters were removed from the aptamer variants BB10 and BB16 (Table 2). The results revealed that the further aptamer variants BB10-6f, BB10-10f, BB10-2r, BB10-9r, BB16-11f and BB16-13f showed higher binding affinity for $B$. breve than their corresponding aptamer variants $\mathrm{BB} 10$ and BB16 respectively, while the further truncated variants BB10-14f, BB10-13r, BB16-15f and BB16-8r

Table 2 Truncated aptamer variants

\begin{tabular}{|c|c|c|}
\hline Name & Sequence $\left(5^{\prime} \rightarrow 3^{\prime}\right)$ & Length (nt) \\
\hline BB2 & CCGGGCAGCGGTCAATGCCGCACCTTCCATATGATCGGGG & 40 \\
\hline BB10 & GGCCCCCTGCCTGCCAAAAAGGTGTTGCCAGGTTGGCGGC & 40 \\
\hline BB10-6f & GGCCCCCTGCCTGCCAAAAAGGTGTTGCCAGGTT & 34 \\
\hline BB10-10f & GGCCCCCTGCCTGCCAAAAAGGTGTTGCCA & 30 \\
\hline BB10-14f & GGCCCCCTGCCTGCCAAAAAGGTGTT & 26 \\
\hline BB10-2r & CCCCCTGCCTGCCAAAAAGGTGTTGCCAGGTTGGCGGC & 38 \\
\hline BB10-9r & CCTGCCAAAAAGGTGTTGCCAGGTTGGCGGC & 31 \\
\hline BB10-13r & CCAAAAAGGTGTTGCCAGGTTGGCGGC & 27 \\
\hline BB16 & CTCCCAGGCCGTTGGGGCGTTGCCTGCGTGCACCCGGGGC & 40 \\
\hline BB16-11f & CTCCCAGGCCGTTGGGGCGTTGCCTGCGT & 29 \\
\hline BB16-13f & CTCCCAGGCCGTTGGGGCGTTGCCTGC & 27 \\
\hline BB16-15f & CTCCCAGGCCGTTGGGGCGTTGCCT & 25 \\
\hline BB16-8r & CCGTTGGGGCGTTGCCTGCGTGCACCCGGGGC & 32 \\
\hline
\end{tabular}




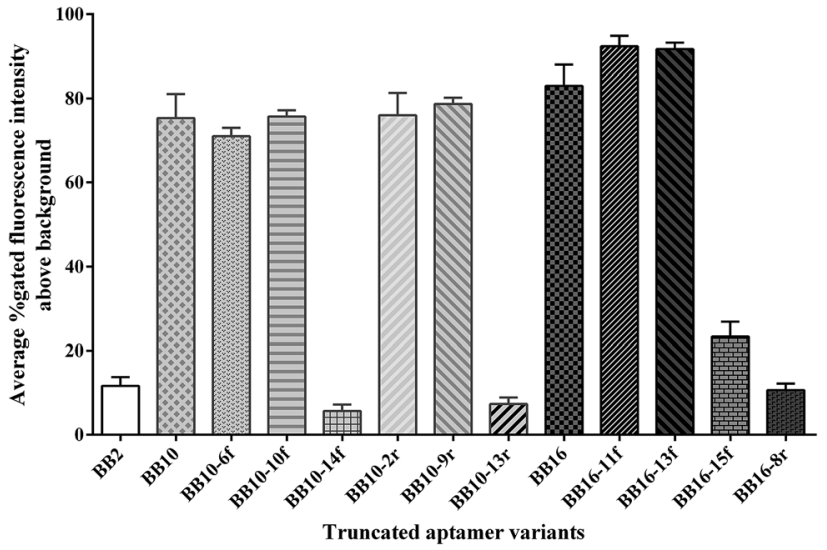

Fig. 3 Binding affinity of the truncated aptamer variants for the target $B$. breve. Binding abilities of these aptamer variants to $B$. breve were analyzed by flow cytometric.

demonstrated lower binding ability to $B$. breve compared to their corresponding aptamer variants (Fig. 3).

Taken together the results of the truncation experiments, the further truncated variants BB16-11f manifested highest binding affinity for its target $B$. breve, with the values of gated fluorescence intensity above the background about $92 \%$, in all the aptamer variants. In the flow cytometric analysis, we did not approach $100 \%$ of aptamer-labeled B. breve cells, which could be explained in part by variation in target marker expression in the bacterial cell populations on the basis of different growth phase. This phenomenon has been demonstrated when wholebacterium SELEX was used to select ssDNA aptamers targeted to S. aureus. $^{33}$

Furthermore, Fig. 4D showed a binding saturation curve from the flow cytometric analysis of the fluorescently labeled aptamer BB16-11f binding to $B$. breve as described above, and the binding dissociation constant $\left(K_{\mathrm{d}}\right)$ of aptamer BB16-11f was measured by nonlinear regression analysis using GraphPad Prism 5.0. The results showed that the $K_{\mathrm{d}}$ value was $18.66 \pm$ $1.41 \mathrm{nM}$ between $B$. breve and the aptamer BB16-11f. Thus, the candidate aptamer BB16-11f was chosen for the further specificity analysis. The binding saturation curve between the aptamer BB16-11f and B. breve and predicted secondary structures were shown in Fig. 4. The conditions input in the prediction of secondary structures were $1 \mathrm{mM} \mathrm{MgCl}_{2}$ and the room temperature.

To determine the specificity of the candidate aptamer BB16$11 \mathrm{f}$ for the target B. breve, FAM-labelled aptamer BB16-11f was
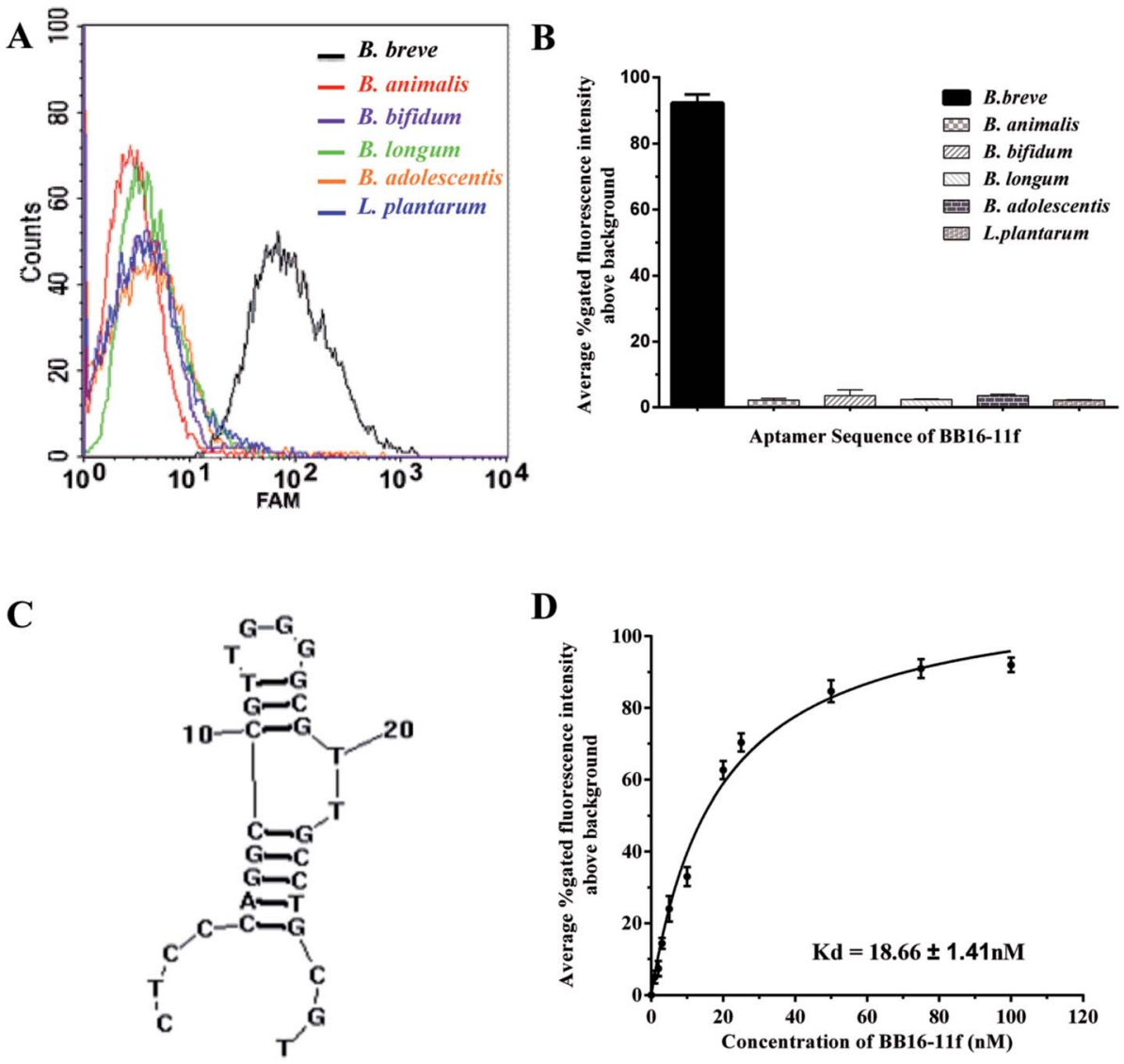

Fig. 4 Binding affinity and specificity of aptamer variant BB16-11f for B. breve. (A) Flow cytometric assays of aptamer BB-16f binding ability to different species of bacteria. (B) Histogram of the percent gated fluorescence intensity above the background for aptamer BB16-11f. (C) Predicted secondary structures of aptamer BB16-11f. The secondary structure of the aptamer BB-11f was predicted by RNA structure 3.0, and the conditions input were $1 \mathrm{mM} \mathrm{MgCl}$ and the room temperature. (D) Binding saturation curve of aptamer BB16-11f to B. breve. A nonlinear regression curve was fit using GraphPad Prism 5.0 as described in text. 
measured against a variety of other bacteria, including $B$. longum, B. bifidum, B. animalis, B. adolescentis and L. plantarum. As shown in Fig. 4, the aptamer BB16-11f showed preferential binding affinity for $B$. breve over the other bacteria tested. This preferential binding ability demonstrated the excellent specificity between the aptamer BB16-11f and the target $B$. breve. Therefore, the aptamer BB16-11f was selected for the subsequent practical application.

\section{The calibration curve of $B$. breve detection}

To prove the potential application of the candidate aptamer BB16-11f, the aptamer/target/aptamer sandwich type was developed. In the experiment, when the reaction of the HRP and TMB- $\mathrm{H}_{2} \mathrm{O}_{2}$ was terminated with $\mathrm{H}_{2} \mathrm{SO}_{4}$, the produced di-imine displayed a yellow color, with an absorbance peak at $450 \mathrm{~nm}$. The results shown in Fig. 5 revealed that the absorbance at $450 \mathrm{~nm}$ ranged from 0.512 to 0.746 when the concentrations of B. breve was in the range from $10^{3} \mathrm{cfu} \mathrm{mL}^{-1}$ to $10^{7} \mathrm{cfu} \mathrm{mL}^{-1}$, and a good linear relationship was determined from the data between the OD values at $450 \mathrm{~nm}$ and the amounts of $B$. breve with a regression coefficient of 0.98 . The limit of detection of the proposed method was estimated to be $1000 \mathrm{cfu} \mathrm{mL}^{-1}$.

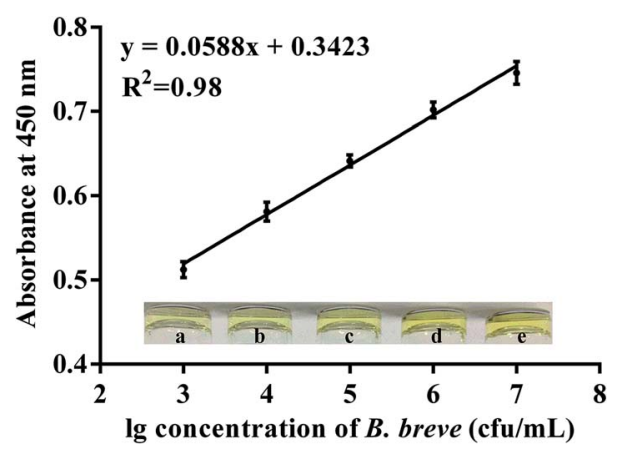

Fig. 5 The calibration curve between the intensity of the signals and the concentrations of $B$. breve. The absorbance was determined at $450 \mathrm{~nm}$ by the microplate reader. Insert: the color is gradually darker from a to e when the concentrations of $B$. breve changed from $10^{3} \mathrm{cfu}$ $\mathrm{mL}^{-1}$ to $10^{7}$ cfu $\mathrm{mL}^{-1}$.

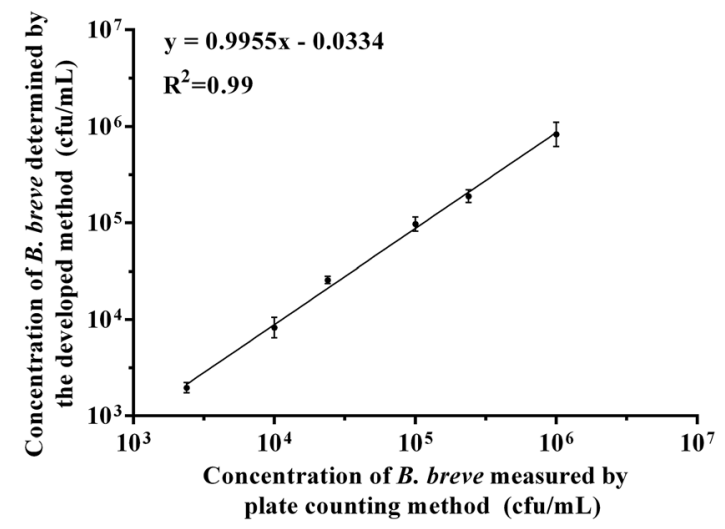

Fig. 6 The relationship between the developed method and plate counting method in detecting $B$. breve in milk samples.

\section{Method validation in milk samples}

To verify the feasibility of colorimetric assay for practical application, we used the developed method to detect $B$. breve in milk, and the plate counting method was also used as control in the study. As shown in Fig. 6, we detected six different concentrations of $B$. breve ranging from $10^{3} \mathrm{cfu} \mathrm{mL}^{-1}$ to $10^{6} \mathrm{cfu}$ $\mathrm{mL}^{-1}$, and the results indicated that the developed method had a good coherence with the plate counting method $\left(R^{2}=0.99\right)$. Therefore, the colorimetric detection method based on the aptamer BB16-11f can be used for the detection of real samples.

\section{Conclusion}

In summary, the ssDNA aptamer BB16-11f with high-affinity binding and excellent specificity for $B$. breve was identified through a whole-bacterium SELEX process and the truncated experiments. The estimated $K_{\mathrm{d}}$ value was $18.66 \pm 1.41 \mathrm{nM}$ between the aptamer BB16-11f and $B$. breve. Furthermore, we established a colorimetric assay which did not rely on expensive instrumentation for the detection of $B$. breve based on the aptamer BB16-11f, and the detection method developed is simple and could be adopted to detect $B$. breve cells as low as $1000 \mathrm{cfu} \mathrm{mL}^{-1}$. In addition, this developed method was also successfully used to detect $B$. breve in milk samples. Therefore, the high-throughput colorimetric assay has been successfully used for detection of $B$. breve.

\section{Acknowledgements}

This work was supported by the Program of National Natural Science Foundation for the Youth of China (No. 31501454), the Program for Changjiang Scholars and Innovative Research Team in University (IRT1249), the Program of Collaborative innovation center of food safety and quality control in Jiangsu Province, and the Program of Introducing Talents of Discipline to Universities (B07029).

\section{References}

1 C. Stanton, G. Gardiner, H. Meehan, K. Collins, G. Fitzgerald, P. B. Lynch and R. P. Ross, Am. J. Clin. Nutr., 2001, 73, 476483.

2 T. Fujii, Y. Ohtsuka, T. Lee, T. Kudo, H. Shoji, H. Sato, S. Nagata, T. Shimizu and Y. Yamashiro, J. Pediatr. Gastroenterol. Nutr., 2006, 43, 83-88.

3 I. Aloisio, C. Santini, B. Biavati, G. Dinelli, A. Cencič, W. Chingwaru, L. Mogna and D. Di Gioia, Appl. Microbiol. Biotechnol., 2012, 96, 1561-1576.

4 L. Mogna, M. Del Piano and G. Mogna, J. Clin. Gastroenterol., 2014, 48, S37-S39.

5 S. G. Jeon, H. Kayama, Y. Ueda, T. Takahashi, T. Asahara, H. Tsuji, N. M. Tsuji, H. Kiyono, J. S. Ma, T. Kusu, R. Okumura, H. Hara, H. Yoshida, M. Yamamoto, K. Nomoto and K. Takeda, PLoS Pathog., 2012, 8, e1002714. 
6 B. Zheng, J. van Bergenhenegouwen, S. Overbeek, H. J. G. van de Kant, J. Garssen, G. Folkerts, P. Vos, M. E. Morgan and A. D. Kraneveld, PLoS One, 2014, 9, e95441.

7 M. Klemenak, J. Dolinšek, T. Langerholc, D. Di Gioia and D. Mičetić-Turk, Dig. Dis. Sci., 2015, 60, 3386-3392.

8 Y. Li, T. Shimizu, A. Hosaka, N. Kaneko, Y. Ohtsuka and Y. Yamashiro, Pediatr. Int., 2004, 46, 509-515.

9 Y. Shimakawa, S. Matsubara, N. Yuki, M. Ikeda and F. Ishikawa, Int. J. Food Microbiol., 2003, 81, 131-136.

10 A. Picot and C. Lacroix, Int. Dairy J., 2004, 14, 505-515.

11 L. Masco, G. Huys, E. De Brandt, R. Temmerman and J. Swings, Int. J. Food Microbiol., 2005, 102, 221-230.

12 G. Mazzola, I. Aloisio, B. Biavati and D. Di Gioia, LWT-Food Sci. Technol., 2015, 64, 727-734.

13 M. Gueimonde, S. Delgado, B. Mayo, P. Ruas-Madiedo, A. Margolles and C. G. de los Reyes-Gavilán, Food Res. Int., 2004, 37, 839-850.

14 M. C. Collado, Y. Moreno, J. M. Cobo and M. Hernández, Eur. Food Res. Technol., 2006, 222, 112-117.

15 J. M. T. Hamilton-Miller, S. Shah and J. T. Winkler, Publ. Health Nutr., 1999, 2, 223-229.

16 R. Temmerman, L. Masco, T. Vanhoutte, G. Huys and J. Swings, Appl. Environ. Microbiol., 2003, 69, 6380-6385.

17 G. Klein, A. Pack, C. Bonaparte and G. Reuter, Int. J. Food Microbiol., 1998, 41, 103-125.

18 E. Vlková, J. Nevoral, B. Jencikova, J. Kopečný, J. Godefrooij, I. Trojanová and V. Rada, J. Microbiol. Methods, 2005, 60, 365-373.

19 R. M. Satokari, E. E. Vaughan, H. Smidt, M. Saarela, J. Mättö and W. M. de Vos, Syst. Appl. Microbiol., 2003, 26, 572-584.

20 T. Takada, K. Matsumoto and K. Nomoto, J. Microbiol. Methods, 2004, 58, 413-421.

21 T. Matsuki, K. Watanabe, J. Fujimoto, Y. Kado, T. Takada, K. Matsumoto and R. Tanaka, Appl. Environ. Microbiol., 2004, 70, 167-173.

22 P. S. Langendijk, F. Schut, G. J. Jansen, G. C. Raangs, G. R. Kamphuis, M. H. Wilkinson and G. W. Welling, Appl. Environ. Microbiol., 1995, 61, 3069-3075.

23 E. Torres-Chavolla and E. C. Alocilja, Biosens. Bioelectron., 2009, 24, 3175-3182.
24 E. C. Alocilja and S. M. Radke, Biosens. Bioelectron., 2003, 18, 841-846.

25 A. D. Ellington and J. W. Szostak, Nature, 1990, 346, 818-822.

26 C. Tuerk and L. Gold, Science, 1990, 249, 505-510.

27 S. M. Nimjee, C. P. Rusconi and B. A. Sullenger, Annu. Rev. Med., 2005, 56, 555-583.

28 A. B. Iliuk, L. Hu and W. A. Tao, Anal. Chem., 2011, 83, 44404452.

29 C. L. A. Hamula, J. W. Guthrie, H. Zhang, X. F. Li and X. C. Le, Trends Anal. Chem., 2006, 25, 681-691.

30 S. M. Shamah, J. M. Healy and S. T. Cload, Acc. Chem. Res., 2008, 41, 130-138.

31 F. Chen, J. Zhou, F. Luo, A. B. Mohammed and X. L. Zhang, Biochem. Biophys. Res. Commun., 2007, 357, 743-748.

32 C. L. A. Hamula, H. Zhang, L. L. Guan, X. F. Li and X. C. Le, Anal. Chem., 2008, 80, 7812-7819.

33 X. Cao, S. Li, L. Chen, H. Ding, H. Xu, Y. Huang, J. Li, N. Liu, W. Cao, Y. Zhu, B. Shen and N. Shao, Nucleic Acids Res., 2009, 37, 4621-4628.

34 H. P. Dwivedi, R. D. Smiley and L. A. Jaykus, Appl. Microbiol. Biotechnol., 2010, 87, 2323-2334.

35 C. L. A. Hamula, X. C. Le and X. F. Li, Anal. Chem., 2011, 83, 3640-3647.

36 N. Duan, S. Wu, X. Chen, Y. Huang, Y. Xia, X. Ma and Z. Wang, J. Agric. Food Chem., 2013, 61, 3229-3234.

37 L. Barthelmebs, J. Jonca, A. Hayat, B. Prieto-Simon and J. L. Marty, Food Control, 2011, 22, 737-743.

38 J. H. Park, Y. S. Cho, S. Kang, E. J. Lee, G. H. Lee and S. S. Hah, Anal. Chem., 2014, 462, 10-12.

39 R. Aimaiti, L. Qin, T. Cao, H. Yang, J. Wang, J. Lu, X. Huang and Z. Hu, Appl. Microbiol. Biotechnol., 2015, 99, 9073-9083.

40 M. Jing and M. T. Bowser, Anal. Chim. Acta, 2011, 686, 9-18.

41 D. Shangguan, Z. Tang, P. Mallikaratchy, Z. Xiao and W. Tan, ChemBioChem, 2007, 8, 603-606.

42 L. Cerchia, F. Ducongé, C. Pestourie, J. Boulay, Y. Aissouni, K. Gombert, B. Tavitian, V. de Franciscis and D. Libri, PLoS Biol., 2005, 3, 0697-0704.

43 D. Shangguan, Y. Li, Z. Tang, Z. C. Cao, H. W. Chen, P. Mallikaratchy, K. Sefah, C. J. Yang and W. Tan, Proc. Natl. Acad. Sci. U. S. A., 2006, 103, 11838-11843.

44 S. D. Jayasena, Clin. Chem., 1999, 45, 1628-1650. 